Article

\title{
Towards Understanding the Raman Spectrum of Graphene Oxide: The Effect of the Chemical Composition
}

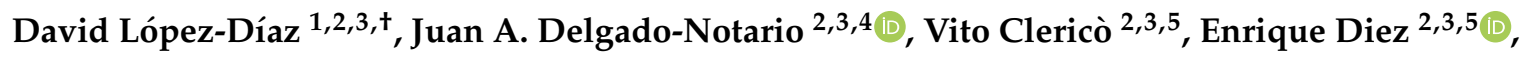 \\ María Dolores Merchán 1,2,3 (D) and María Mercedes Velázquez 1,2,3,*(D) \\ 1 Departament of Physical Chemistry, Faculty of Chemical Sciences, University of Salamanca, \\ E37008 Salamanca, Spain; david.lopez@uah.es (D.L.-D.); mdm@usal.es (M.D.M.) \\ 2 Group of Nanotechnology, Faculty of Physics and Faculty of Chemical Sciences, University of Salamanca, \\ E37008 Salamanca, Spain; juanandn@usal.es (J.A.D.-N.); vito_clerico@usal.es (V.C.); enrisa@usal.es (E.D.) \\ 3 NANOLAB-USAL, Laboratory of Nanoelectronics and Nanomaterials, University of Salamanca, \\ E37008 Salamanca, Spain \\ 4 Department of Applied Physics, Faculty of Sciences, University of Salamanca, E37008 Salamanca, Spain \\ 5 Department of Fundamental Physics, Faculty of Sciences, University of Salamanca, E37008 Salamanca, Spain \\ * Correspondence: mvsal@usal.es \\ † Current Address: Departamento de Química Analítica, Química Física e Ingeniería Química, \\ Universidad de Alcalá, 28871 Alcalá de Henares, Spain.
}

Received: 17 April 2020; Accepted: 27 May 2020; Published: 29 May 2020

check for updates

\begin{abstract}
Raman spectroscopy is a technique widely used to detect defects in semiconductors because it provides information of structural or chemical defects produced in its structure. In the case of graphene monolayer, the Raman spectrum presents two bands centered at $1582 \mathrm{~cm}^{-1}(\mathrm{G}$ band) and $2700 \mathrm{~cm}^{-1}$ (2D band). However, when the periodic lattice of graphene is broken by different types of defects, new bands appear. This is the situation for the Raman spectrum of graphene oxide. It is well established that the existence of these bands, the position and the intensity or width of peaks can provide information about the origin of defects. However, in the case of the graphene oxide spectrum, we can find in the literature several discrepant results, probably due to differences in chemical composition and the type of defects of the graphene oxide used in these studies. Besides, theoretical calculations proved that the shape of bands, intensity and width, and the position of graphene oxide Raman spectrum depend on the atomic configuration. In the current work, we will summarize our current understanding of the effect of the chemical composition on the Raman spectrum of graphene oxide. Finally, we apply all this information to analyze the evolution of the structure of graphene oxide during the thermal annealing of the heterostructures formed by graphene oxide sandwiches in a hexagonal boron nitride.
\end{abstract}

Keywords: graphene oxide; hexagonal boron nitride; Raman; heterostructures

\section{Introduction}

Graphene oxide (GO) is a material derivate of graphene, which is produced by the oxidation of different types of graphite or carbon nanofibers. From the structural point of view, it has a similar hexagonal carbon network to graphene; however, it contains different types of oxygen functional groups. The O-functionalization and the existence of structural defects on the basal plane due to the strong oxidation process remarkably reduce the excellent optoelectronic properties of graphene. Nevertheless, the O-groups attached at the carbon network allow joining polymers or nanoparticles to build polymer composites [1], a gas sensors [2], photovoltaic cells [3], drug delivery vectors [4], or for 
capturing $\mathrm{CO}_{2}$ [5]. Besides, O-groups provide active adsorption sites to remove heavy metal ions [6] and other pollutants [7]. The possibility of using GO in these applications justifies the interest aroused by this material in recent years. However, each application needs a suitable oxidation degree; therefore, several methods for modulating the $\mathrm{C} / \mathrm{O}$ atomic ratio of $\mathrm{GO}$ have been reported. Chemical and thermal reduction methods are two of the most widely employed. Chemical reducers as hydrazine hydrate or ascorbic acid proved to be good reducers of graphene oxide [8-10], but, chemical reagents often include functional groups of reducer agents, which deteriorate some properties of GO [11,12]. Thermal annealing at high temperature $\left(>750^{\circ} \mathrm{C}\right)$ significantly increases the $\mathrm{C} / \mathrm{O}$ atomic ratio. However, it is necessary as an exhaustive control of the heat rate, because, if the temperature quickly increases, vacancies and other structural defects on the network have been observed [13]. Recently, using the nonequilibrium technique of nanosecond pulsed laser annealing, a high C/O atomic ratio (16) was reached [14]. The properties of GO can be modulated by introducing different functional groups and structural defects. Therefore, identification and quantification of defects are crucial. Raman spectroscopy is a sensitive and nondestructive technique widely used to detect disorders in $C s p^{2}$ systems because its phonon modes provide information about chemical or structural defects. Following the works of Dresselhaus [15-18] and Ferrari [19-22], it was possible to understand the spectrum of graphene. This spectrum consists of two peaks centered at $1582 \mathrm{~cm}^{-1}$ ( $G$ band) and $2700 \mathrm{~cm}^{-1}\left(\mathrm{G}^{\prime}\right.$ or $2 \mathrm{D}$ band) [23,24]. These bands satisfy the Raman selection rules [9]; however, when the graphene network breaks due to defects, two new bands, D and $\mathrm{D}^{\prime}$, centered at 1350 and $1626 \mathrm{~cm}^{-1}$ appear. These new bands are forbidden, although, they are activated by defects through two mechanisms, single phonon intervalley and intravalley scattering, respectively. In addition, the intensity of the 2D band decreases while broad features attributed to the 2D'and combination bands appear $[15,25]$. In the case of the GO Raman spectrum, the literature presents a great variety of results and discrepancies as well. Some works showed that the intensities of D and G bands are almost the same, while the intensity of the 2D band intensity is too low or is not even considered. Other works reported differences concerning the position and width of the $\mathrm{G}$ band, which are often accompanied by the appearance of new bands attributed to vacancies or Stone-Wales defects [26,27]. Besides, the Raman spectrum of GO has been simulated using ab initio calculations. [28,29]. These calculations showed that in the GO Raman spectrum, the intensity of the $G$ band and the appearance of new bands are related to the local atomic configuration. Based on this theoretical framework, we think that to interpret the Raman spectrum of GO, it is necessary to perform a systematic study of the effects of functional groups on the spectrum. With this objective in mind, in recent years we studied the evolution of the Raman spectrum of GO with the composition and structural defects. Firstly, we selected graphene oxides obtained by oxidation of graphitized carbon nanofibers (GANF ${ }^{\circledR}$ ) followed by thermal annealing [12]. Next, we synthesized different graphene oxides by the oxidation of three types of graphite and two commercial carbon nanofibers. To remove oxidation impurities, we have washed these graphene oxides with alkali resulting graphene nanosheets with lower oxidation degree values than the non-purified ones [30]. These two routes of synthesis allow us to modulate the O-groups attached to the basal plane and the structural defects of the network in a systematic way. So, to analyze the effect of the oxidation degree minimizing the effects of heterogeneity, we selected graphene oxides obtained by the oxidation of carbon nanofibers thermally reduced between 100 and $800^{\circ} \mathrm{C}$. Furthermore, to study the effect of structural heterogeneities, we selected graphene oxides synthesized by the same oxidation route but using different starting materials and purification methods. This methodology allows us to modify both chemical compositions and the structural defects.

In the current work, we will summarize our current understanding of the Raman spectrum of graphene oxide and present the correlations between the position, width, intensity of different bands and the chemical composition of GO, and the type of structural defects previously reported by us [30]. We expect that these correlations can be applied to obtain structural information of graphene oxide by recording its Raman spectrum in situations where it is not possible to analyze the chemical composition by widely used techniques, such as organic elemental analysis or X-ray photoelectron 
spectroscopy, XPS. An example is the manufacture of heterostructures. Heterostructures formed by interfacing two different semiconductors play an important role in the semiconductor industry since they are an essential component of electronic devices. Some 2D materials, such as graphene, hexagonal boron nitride $(h-B N)$, or transition metal dichalcogenides (TMDs) present a unique optical bandgap, interact strongly with light, and present a large interfacial area. For these reasons, they are considered excellent components of electronic devices providing a wide range of materials from semiconductors to insulators. In graphene-based heterostructures, the most common stacking procedures are mechanical exfoliation and chemical vapor deposition, CVD [31]. These methodologies produce defects in the materials decreasing the quality of the devices [32], therefore, it is necessary to have a technique capable of detecting these defects directly in the heterostructure. To enhance the production of 2D materials, graphene is synthesized from graphene oxide followed by thermal reduction. In this method, graphene oxide is transferred onto the solid substrate and then thermally reduced. Thermal reduction at high temperature annealing $\left(>750^{\circ} \mathrm{C}\right)$ can remove almost all O-groups [13], although, if the temperature is quickly increased, some vacancies in the network are created decreasing the quality of the heterostructure. [11,12]. Based on this framework, in the current work we will use the reported correlations between the features of the GO Raman spectrum and the chemical composition and origin of structural defects of graphene oxide to study the effect of annealing on the composition and defects of heterostructures of GO sheets sandwiched between hexagonal boron nitride flakes. The presence of $h-B N$ guarantees more thermal stability and it is inert against many chemicals. These properties are useful in applications where thermal stability is a challenge, as recently demonstrated for high temperature $\mathrm{RCO} / \mathrm{h}-\mathrm{BN}$ based Li-ion batteries [33].

\section{Materials and Methods}

\subsection{Reagent and Graphene Oxide Synthesis}

Graphene oxide was obtained using the oxidation procedure reported by Hummers slightly modified by our group $[10,30,34]$ and natural graphite flakes as a starting material. To separate high-quality sheets from GO solutions we filtrate the GO dispersions through sieves of a mesh size of $0.425,0.090$, and $0.020 \mathrm{~mm}$ [35]. The chemical composition of GO was determined by XPS and the C/O value was 1.46 [35]. The percentage of $\mathrm{Csp}^{2}$ found was $64 \pm 1.9$.

The reagents employed for preparing heterostructures, anisole, acetone, and isopropanol, poly (propylene carbonate) were purchased from Sigma Aldrich (St. Louis, MO, USA) and SYLGARD ${ }^{\mathrm{TM}} 184$ Silicone Elastomer kit from Dow Corning (Midland, MI, USA). Natural graphite flakes (99.02 fixed C) used as starting material to synthesize graphene oxides was supplied by Qingdao Super Graphite Co. (Qingdao, China) and hexagonal boron nitride $(h-B N)$ crystals were purchased from HQ graphene (Groningen, The Netherlands). Graphene oxide was mechanically exfoliated with Magic Scotch Tape and $h$-BN crystals were exfoliated with 1009R-6.0 silicone-free adhesive plastic film supplied by Ultron Systems, Inc. (Moorpark, CA, USA).

\subsection{Methods}

X-ray photoelectron spectrum of GO powder was recorded in a VG Escalab 200R spectrometer (Fisons Instruments, Parkton, MD, USA). The excitation source was the $\mathrm{Mg} \mathrm{K} \alpha(h v=1253.6 \mathrm{eV})$ radiation. The equipment uses a hemispherical electron analyzer. XPS spectra were recorded at $20 \mathrm{eV}$ analyzer pass energy. The residual pressure in the analysis chamber remains under a value of $4 \times 10^{-7} \mathrm{~Pa}$.

Functionalization of the surface of Poly (di-methyl-siloxane) substrate was made through an Inductively Coupled Plasma - Reactive Ion Etching (ICP-RIE) model PlasmaPro ${ }^{\circledR}$ Estrelas 100 from Oxford Instruments, Yatton, UK) process in $\mathrm{O}_{2}$ atmosphere. Annealing processes were performed with the system rapid thermal processing (RTP) As-One 100 from ANNEALSYS (Montpellier, France). 
Raman spectra were obtained with a confocal Raman microscope LabRAM HR Evolution spectrometer from Horiba Jobin-Yvon (Kyoto, Japan). Materials were irradiated with light from a solid-state laser at $532 \mathrm{~nm}$. We used a 100× objective (laser spot size $1 \mu \mathrm{m}^{2}$ ). To avoid laser-induced heating, the power of the excitation laser remained below $1 \mathrm{~mW}$. The band centered at $520.7 \mathrm{~cm}^{-1}$ corresponding to $\mathrm{Si}$ was used to calibrate the spectrometer. The spatial and the spectral resolution were $0.5 \mu \mathrm{m}$ and $2 \mathrm{~cm}^{-1}$, respectively and the acquisition time at each point was $3 \mathrm{~s}$. We recorded the Raman spectrum at least in five different regions of the heterostructure and each spectrum showed in figures was the average of all spectra. The diffraction grating used in all spectra was $1800 \mathrm{gr} \mathrm{mm}^{-1}$.

\section{Results}

\subsection{Graphene Oxide Raman Spectrum}

Raman spectroscopy is a non-destructive technique widely used to investigate carbon nanomaterials. However, the analysis and interpretation of the Raman spectrum of graphene oxide are lagging compared to the information gained for other carbon nanomaterials such as graphene or carbon nanotubes. To deepen the interpretation of the bands of the Raman spectrum of GO, in recent years we carried out a systematic study of the effect of functionalization and structure of GO on its Raman spectrum. To achieve this goal, we used two different strategies; in the first one, to analyze the effect of the chemical composition minimizing heterogeneity effects, we selected graphene oxides obtained by oxidation of carbon nanofibers and annealed between 100 and $800{ }^{\circ} \mathrm{C}$. In the second strategy, to study the effect of structural heterogeneities, we selected graphene oxides synthesized by the same oxidation route but using different starting materials. In this way, we managed to modify the functional groups attached at the basal plane and the structural defects of graphene oxides.

Our results showed that the Raman spectra of these materials presented D and G bands centered at 1350 and $1585 \mathrm{~cm}^{-1}$ respectively, and the second-order spectrum above $2900 \mathrm{~cm}^{-1}$. In addition, new bands centered at $1610 \mathrm{~cm}^{-1}$ ( $\mathrm{D}^{\prime}$ band), $1530 \mathrm{~cm}^{-1}$ ( $\mathrm{D}^{\prime \prime}$ band), and $1150 \mathrm{~cm}^{-1}$ ( $\mathrm{D}^{*}$ band) also appeared. Bands D and $\mathrm{D}^{\prime}$ appear when defects break the periodic lattice of graphene [36]. To interpret the origin of $\mathrm{D}^{\prime \prime}$ and $\mathrm{D}^{*}$ bands, we combined organic elemental analysis, X-ray diffraction measurements, and Raman spectroscopy [12]. Our results confirmed that the D" band is related to amorphous phases [37] since its intensity and width decreased with the crystallinity. Band $\mathrm{D}^{*}$ has been observed in several carbonaceous materials, in diamonds it was related to trans polyacetylene groups [38], and in soot and other materials it was related to disordered graphitic lattice provided by the existence of $s p^{3}$ bonds [39-41]. In graphene oxides synthesized from carbon nanofibers, we found correlations between both the intensity and the maximum position of the $\mathrm{D}^{*}$ band and the oxidation degree. Thus, the intensity decreased when the oxygen content decreased, while the maximum position shifted to lower wavenumber values when the oxygen content increased [12]. This behavior justifies that in graphene oxides, the $\mathrm{D}^{*}$ band could be related with disorder graphitic lattice due to the presence of Csp ${ }^{3}$ bonds.

On the other hand, it is well established that the $I_{D} / I_{G}$ ratio is related to the inverse of the lateral crystallite size, $L_{a}$, through the Tuinstra-Koenig correlation [36]. This correlation has been extensively used to estimate the degree of defects in the graphene network [36] and to study the degree of reduction of GO [42]. Therefore, we checked the validity of the Tuinstra-Koenig formalism in these graphene oxides. Our results proved that the variation of the $I_{D} / I_{G}$ ratio with the $L_{a}$ values calculated from XRD measurements cannot be interpreted according to the Tuinstra-Koenig correlation, but it fit very well to the Cuesta's formalism [12,43]. Since the Cuesta's model was proposed to interpret Raman measurements of carbon-based materials with a certain disorder degree, we concluded that GO obtained from carbon nanofiber and thermally annealed presents a relatively degree of disorder [12]. We analyzed the origin of it below.

The second strategy consisted of the synthesis of graphene oxides using the same oxidation procedure and different starting materials [30]. Using this methodology, it was possible to obtain 
graphene oxides with different O-groups and structural defects [30]. After the synthesis and characterization of different graphene oxides, their Raman spectra were recorded to analyze the effect of the functional groups and structural defects on the Raman spectrum of GO. The first-order Raman spectra were split in five functions assigned to $\mathrm{D}, \mathrm{G}, \mathrm{D}^{\prime}, \mathrm{D}^{\prime \prime}$, and $\mathrm{D}^{*}$ bands. Among these bands, we found three peaks centered at 2690,2930, and $3190 \mathrm{~cm}^{-1}$, respectively. The peak centered at $2690 \mathrm{~cm}^{-1}$ was ascribed to the 2D band [16], but the assignation of the other peaks were controversial. To assign these bands, in previous work [30], we have plotted the frequency of the 2D band against twice the frequency of the $\mathrm{D}$ band, the frequency of the combination $\mathrm{D}+\mathrm{D}^{\prime}$ band vs. the sum of the frequency of $\mathrm{D}$ and $\mathrm{D}^{\prime}$ bands and finally, the frequency of the 2D' band against twice the frequency of the $\mathrm{D}^{\prime}$ band, respectively. The data fit very well allowing us to assign unambiguously these peaks to $2 \mathrm{D}, \mathrm{D}+\mathrm{D}^{\prime}$, and 2D' bands, respectively.

Our results demonstrated that the $I_{D} / I_{G}$ ratio and the frequency of the maximum of the $2 \mathrm{D}$ and $\mathrm{D}+\mathrm{D}^{\prime}$ bands linearly depended on the percentage of $\mathrm{C} s p^{2}$ obtained by XPS measurements [30]. Consequently, they can be used to estimate the percentage of $C s p^{2}$ of graphene oxides. This is consistent with results reported by other authors who found correlations between the area of 2D and the combination band with the electrical resistance of reduced graphene oxide and, consequently, with the degree of reduction of GO [44].

To interpret the evolution of $\mathrm{D}$ and $\mathrm{D}^{\prime}$ bands with structural defects, we used the double-resonance mechanism proposed to interpret the Raman spectrum of defective graphene [45]. The model considers that the number of defects is related to the electron lifetime. Accordingly, the intensity of both, the D and $\mathrm{D}^{\prime}$ bands is proportional to the concentration and type of defects, i.e., grain boundaries, vacancies, and $s p^{3}$ hybridization, respectively [45]. To analyze this dependence, two different situations must be considered, graphene materials with low and high of disorder degrees, respectively. The classification introduced by Ferrari considers that the $I_{D} / I_{G}$ ratios below 3.5, (at $2.41 \mathrm{eV}$ ) correspond to materials with a low degree of disorder, while $I_{D} / I_{G}$ [46] ratios above 3.5 correspond to highly disordered materials. In the case of a low disorder degree, since the intensities of the $\mathrm{D}$ and $\mathrm{D}^{\prime}$ peaks were proportional to the defect concentration, they were proportional to each other. Besides, results from ab initio calculations for low-defect graphenes showed that the $\mathrm{I}_{\mathrm{D}^{\prime}} / \mathrm{I}_{\mathrm{D}}$ value for graphene with vacancies was 0.14 , while graphene with $\mathrm{sp}^{3}$ defects and grain boundaries had $I_{D^{\prime}} / I_{D}$ values of 0.08 and 0.29 , respectively $[45,47,48]$. According to $I_{D} / I_{G}$ values found for graphene oxides synthesized by oxidation of different starting materials present low-disorder degree and from the $I_{D^{\prime}} / I_{D}$ calculated, we showed that vacancy-like defects predominate in larger GO sheets (diameter $>400 \mathrm{~nm}$ ) while the defects due to $\mathrm{C} s p^{3}$ predominate in small sheets of a diameter around $100 \mathrm{~nm}$ [30].

In the current work, we analyzed the $I_{D^{\prime}} / I_{D}$ values calculated for graphene oxides synthesized by oxidation of carbon nanofibers $\mathrm{GANF}^{\circledR}$ followed by thermal annealing. It is necessary to consider that according to the Ferrari's classification these materials present $I_{D} / I_{G}$ ratios below 3.5 and consequently, low disorder degree, therefore a linear dependence between $A_{D^{\prime}} / A_{G}$ and $A_{D} / A_{G}$ is expected. This behavior was confirmed by plotting the relative intensity of the $\mathrm{D}^{\prime}$ band, $A_{D^{\prime}} / A_{G}$, against the relative intensity of the $\mathrm{D}$ band, $A_{D} / A_{G}$, Figure 1 . We always prefer to express the relative intensity of bands in terms of the integrated areas instead the intensity since the area under a band represents the probability of the scattering process [46]. Data in Figure 1 were taken from reference [12].

Figure 1 clearly shows that the $A_{D^{\prime}} / A_{G}$ linearly depended on the $A_{D} / A_{G}$ values. Furthermore, the $A_{D^{\prime}} / A_{D}$ value calculated from the linear fit of experimental data was 0.283 . This value agrees very well with the theoretical value reported for grain boundary defects, $0.29[45,47,48]$. This means that in the case of thermal annealed of graphene oxides grain boundaries predominated against vacancies or $\mathrm{sp}^{3}$ defects. The existence of grain boundaries on the graphene oxide sheets synthesized from GANF ${ }^{\circledR}$ could be the reason of the disorder degree detected in these materials commented above.

Finally, we have previously reported that the $I_{D} / I_{G}$ ratio and the position of the maximum of the $2 \mathrm{D}$ and the combination band $\mathrm{D}+\mathrm{D}^{\prime}$ linearly depend on the percentage of $\mathrm{C} s p^{2}$ [30]. Consequently, these correlations could be used to estimate the $\mathrm{Csp}^{2}$ percentage in those situations where it is difficult 
to determine it. An example of this situation is the analysis of the evolution of the degree of oxidation with thermal annealing of a sheet inserted between hexagonal boron nitride $(h-B N)$ flakes, forming a heterostructure. We applied these correlations to obtain structural information of heterostructures of graphene oxide sandwiches between $h-B N$ sheets.

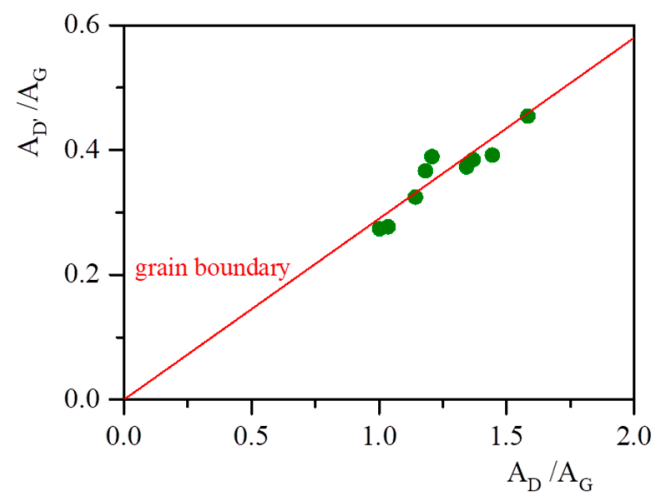

Figure 1. Variation of the relative intensity of the $\mathrm{D}^{\prime}$ band with the relative intensity of the $\mathrm{D}$ band for different graphene oxides obtained from $\mathrm{GANF}^{\circledR}$ carbon nanofibers followed by thermal annealing. The relative intensities are expressed in terms of area under bands. The line was calculated according to theoretical model for grain boundary defects.

\subsection{Aplication of Raman Spectroscopy to the Structural Characterization of $h-B N-G O-h$-BN Heteroestructures.}

To construct $h-B N-G O-h-B N$ heterostructures, graphene oxide and hexagonal boron nitride flakes were obtained by the mechanical exfoliation technique. Firstly, $h-B N$ flakes were obtained by the standard mechanical exfoliation method [48] on a $290 \mathrm{~nm}$ thick $\mathrm{SiO}_{2}$ thermally grown on a 4-inch $\mathrm{Si}$ wafer, Figure 2a. When the same procedure was used to produce GO flakes, we found that the exfoliated sheets broke in flakes too smaller to manufacture heterostructures. To solve this problem, we consider the possibility of adding a chemical to favor the exfoliation of graphene oxide. It is well established that water can be exfoliated at the industrial level by sonication in water because it weakens bonds between platelets favoring the exfoliation process [49]. Therefore, prior to the mechanical exfoliation, GO crystals were mixed with water and then placed on an adhesive Scotch Tape $^{\mathrm{TM}}$, which was folded several times into itself. This methodology prevents the sheets fracture in too small flakes. Next, it was contacted with a poly (di-methyl-siloxane), PDMS, substrate instead of the conventional $\mathrm{SiO}_{2} / \mathrm{Si}$ substrate. The PDMS was homemade from a 10:1 mix ratio of the base and curing agent of the SYLGARD ${ }^{\mathrm{TM}} 184$ Silicone. The two components were previously mixed in a baker, so we removed all the air bubbles trapped into the polymer applying different steps of pump and vent processes in a vacuum hood [50]. The PDMS is placed in a 4-inch square petri dish and heated at $75^{\circ} \mathrm{C}$ for $2 \mathrm{~h}$. For exfoliation of GO, we cut a piece $(1 \mathrm{~cm} \times 1 \mathrm{~cm})$ of PDMS and the adhesive tape with exfoliated GO was placed on the PDMS stamp and the tape was gently removed, completing the exfoliation of GO. Figure $2 \mathrm{~b}$ shows the image obtained by the optical microscope of GO flakes of different sizes randomly distribute on the PDMS substrate.

Since the PDMS is transparent, GO flakes can be easily identified through it and then transferred into another substrate enabling the staking process and opening a new way on the GO based heterostructures fabrication. The GO based heterostructures were fabricated using a two-step stacking method. First, GO/h-BN heterostructures were fabricated using a method similar to that described by Castellanos et al. [51] where the exfoliated GO on the PDMS substrate is aligned through an optical microscope and $\mathrm{XYZ}$ micromanipulators with the h-BN flake (bottom h-BN) and then transferred to the $h-B N$ substrate. For this process, the alignment was performed through optical microscope and micrometric screws in $(\mathrm{x}, \mathrm{y}, \mathrm{z})$ axis, Figure $2 \mathrm{~d}$. Once the GO is aligned with the bottom $h-B N$ flake, we moved up the substrate with bottom $h-B N$ and, increasing the temperature until $80{ }^{\circ} \mathrm{C}$, we released 
the GO on the bottom $h-B N$. A fast cleaning in acetone and isopropanol is enough to remove the few contaminations due to the first transfer process.
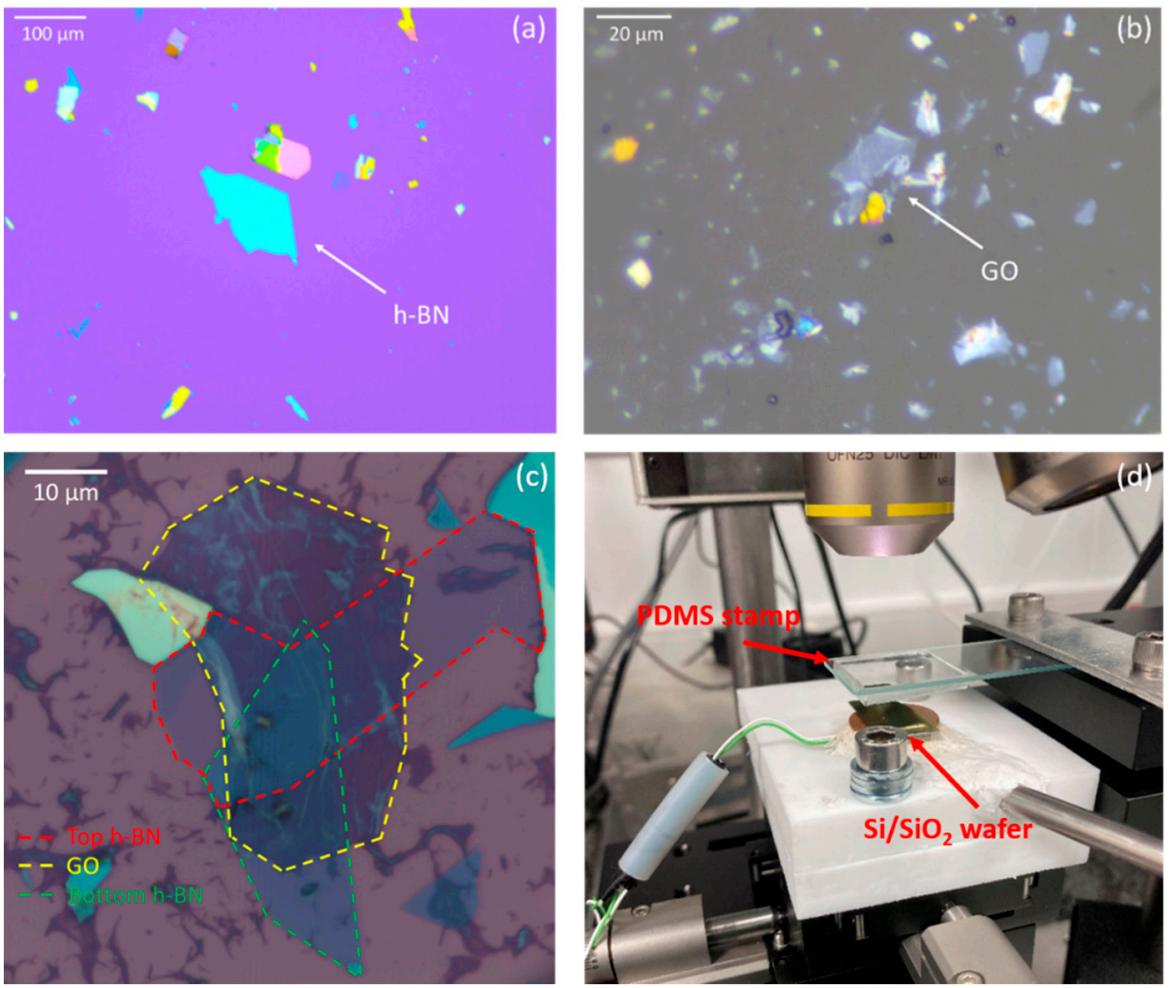

Figure 2. (a) $h-B N$ and (b) GO flakes obtained by mechanical exfoliation. (c) Full $h-B N-G O-h-B N$ heterostructure. (d) Staking system for the fabrication of high-quality 2D heterostructures at USAL-NANOLAB.

Once the heterostructure of $\mathrm{GO}$ on $h-B N$ was obtained, the full heterostructures ( $h$ - $\mathrm{BN} / \mathrm{GO} / h-\mathrm{BN})$ were fabricated by transferring another $h-B N$ flake (top $h$-BN) to the $\mathrm{GO} / h$-BN heterostructure by means of the polymer-based method [52] using poly(propylene carbonate), PPC. For the PPC stamp, we spun the PPC dissolved in anisole at $14 \%$ on a PDMS square of $1 \mathrm{~cm} \times 1 \mathrm{~cm}$ and heated in vacuum at $100{ }^{\circ} \mathrm{C}$ for $15 \mathrm{~min}$. The PDMS surface was previously functionalized through an ICP-RIE process in $\mathrm{O}_{2}$ atmosphere. The stamp of PPC and PDMS was stacked on a microscope glass. First, we picked up the top $h$-BN touching with the stage movement the wafer with $h$-BN and extending the contact area by heating. After turning off the heater, we lowered the stage, picking up the top $h$-BN. Later we aligned and put in touch the top $h$-BN on the PPC/PDMS stamp with the GO/h-BN bottom heterostructure, once a contact area appeared, we increased the temperature at values higher than 100 degrees, melting the PPC film on the $\mathrm{SiO}_{2}$ wafer and obtaining the full heterostructure ( $h$-BN-GO- $h$-BN). Finally, the residual PPC polymer was removed by washing with anisole, acetone, and isopropanol.

After fabrication of the $h$-BN-GO- $h$-BN heterostructure, we recorded the Raman spectra at room temperature and annealed in a nitrogen atmosphere from 100 to $1200{ }^{\circ} \mathrm{C}$. Two illustrative examples corresponding to the Raman spectra of a heterostructure at room temperature and after annealing at $1200^{\circ} \mathrm{C}$ are collected in Figure 3.

It was reported that the position and width of the Raman spectrum of $h$-BN recorded in heterostructures of graphene $/ h$ - $\mathrm{BN}$ remains steady up to $1250^{\circ} \mathrm{C}$ [53]. Therefore, we focus exclusively on variations produced on the graphene oxide after being annealed. All the spectra present three main features centered at $1360 \mathrm{~cm}^{-1}$ (D band), $1585 \mathrm{~cm}^{-1}$ ( $\mathrm{G}$ band), and $2700 \mathrm{~cm}^{-1}$ (2D). The relative intensities of these bands changed after annealing. Thus, the intensity of the $2 \mathrm{D}$ band increased 
after thermal reduction. Besides, the intensity of the $D$ band decreased relative to the intensity of $G$ band during the annealing. These behaviors were characteristic of the reduction of graphene oxide. To quantify changes in the spectra, we split the first-order Raman spectra into three pseudo-Voigt functions and the second-order spectrum into four Lorentzian contributions. These functions were used in previous works $[12,27,30]$. It is interesting to note that $\mathrm{D}^{*}$ and $\mathrm{D}^{\prime \prime}$ bands were too small to be detected by the fitting procedure. This fact indicates that the GO flake sandwiched in the heterostructure presents a relatively high degree of crystallinity and ordered graphitic lattice. After deconvolution, the $I_{D} / I_{G}$ ratio was calculated and its variation with the temperature of annealing is plotted in Figure 4a.

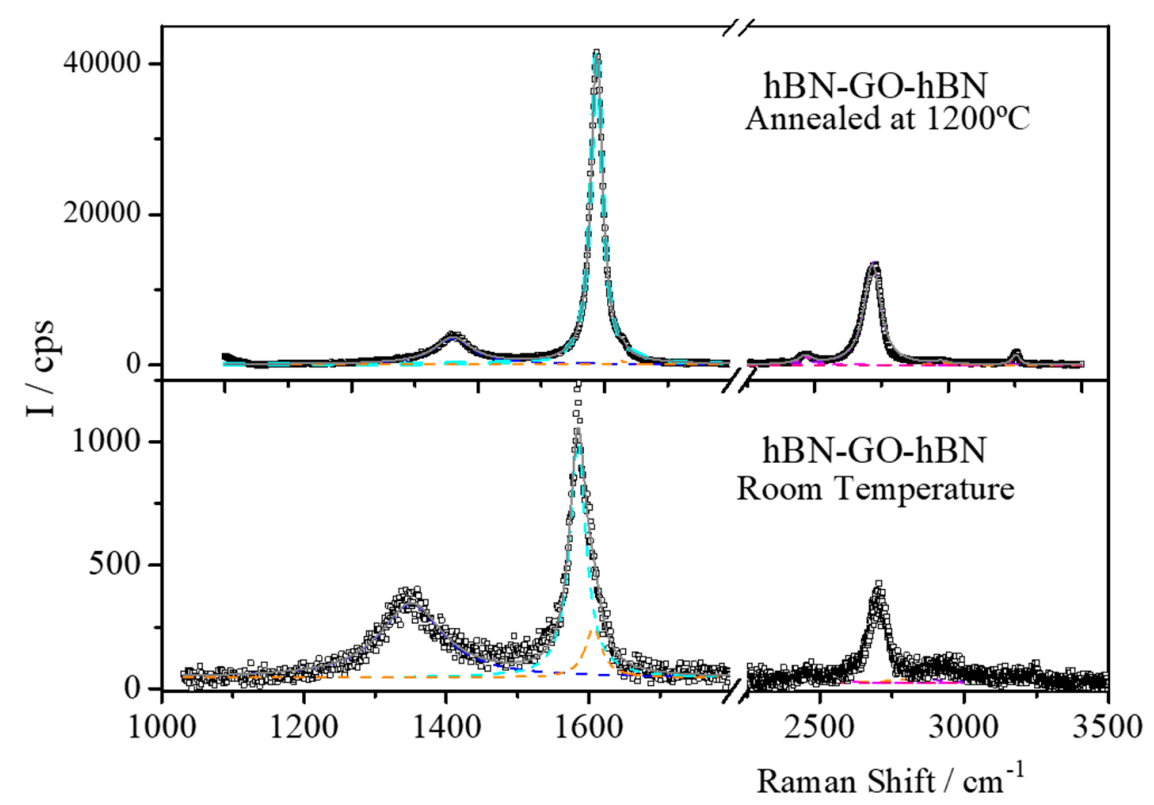

Figure 3. Raman spectra of the $h$-BN-GO- $h$-BN heterostructure (room temperature) and annealed at $1200^{\circ} \mathrm{C}$. Symbols represent experimental spectra; dashed lines represent the functions into which we split the spectrum and solid lines are fits to the data.
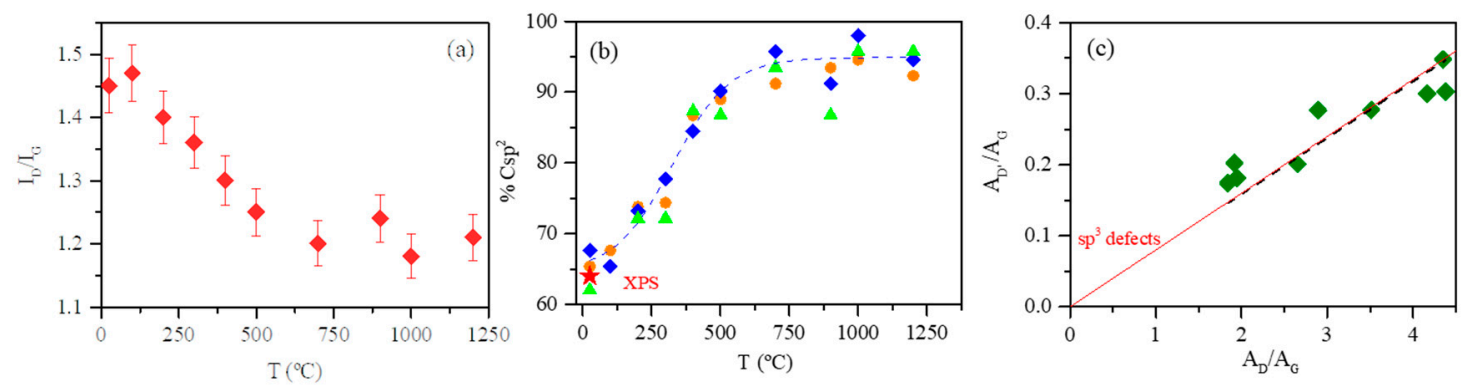

Figure 4. (a) Variation of the $I_{D} / I_{G}$ ratio with temperature for graphene oxides in heterostructures and annealed at between 25 and $1200{ }^{\circ} \mathrm{C}$. (b) Variation of the percentage of $C s p^{2}$ with temperature calculated from the following correlations: $I_{D} / I_{G}$ ratio (circles), frequency of $2 \mathrm{D}$ band (diamonds) and the frequency of the combination band (triangles). Scatter graphs represent experimental data and the dotted line is just a visual guide. (c) Variation of the relative integrated area of the $\mathrm{D}^{\prime}$ band with the relative integrated area of the $\mathrm{D}$ band for graphene oxides in heterostructures annealed at different temperatures. Dotted line is the linear fit of the experimental data and the solid line is calculated according to the theoretical model for $s p^{3}$ defects.

The percentage of $C s p^{2}$ was calculated using the three linear correlations between the $I_{D} / I_{G}$ ratio, the positions of the $2 \mathrm{D}$ and the $\mathrm{D}+\mathrm{D}^{\prime}$ combination band and the $\mathrm{C} s p^{2}$ percentage previously reported [30], Figure $4 \mathrm{~b}$. It is worth noting that the percentage of $C s p^{2}$ calculated from the three correlations agreed very well. Figure $4 \mathrm{~b}$ also presents the $C s p^{2}$ value obtained from the $\mathrm{X}$-ray 
photoelectron spectrum (XPS) of as synthesized GO. As can be seen the value was in excellent agreement with that obtained for the $h$-BN-GO- $h$-BN heterostructure at room temperature calculated from the reported correlations. This is a strong argument to demonstrate the validity of these correlations. Finally, in Figure $4 c$ the relative intensity expressed as the integrated area of $A_{D^{\prime}} / A_{G}$ vs. $A_{D} / A_{G}$ are plotted. The solid line in Figure $4 \mathrm{c}$ was obtained from ab initio calculations for graphene with $s p^{3}$ defects and the dotted line corresponded to the linear fit of the experimental data. As can be seen, both lines coincided with each other, indicating that the $\mathrm{sp}^{3}$ defects predominated in graphene oxide sheets incorporated to the $h$-BN-GO- $h$-BN heterostructure and thermally annealed.

\section{Conclusions}

This paper summarized the reported Raman data of graphene oxides of different chemical compositions and structural defects. The results allowed us to obtain different correlations between the intensity, the position, and the width of the Raman spectrum and the crystallinity, oxidation degree, and structural defects of GO. We used these correlations to study the structural changes produced by thermal annealing of GO sandwiches between hexagonal boron nitride flakes. The correlations between the $I_{D} / I_{G}$ ratio and the frequency of the maximum of $2 \mathrm{D}$ and the combination band $\mathrm{D}+$ $\mathrm{D}^{\prime}$ and the percentage of $\mathrm{C} s p^{2}$ allow calculating the oxidation degree after annealing. It is worth noting that the percentage of $\mathrm{C} s p^{2}$ value of GO at room temperature very well agreed with the value found using XPS, this fact validates the reported correlations. Besides, the $A_{D^{\prime}} / A_{G}$ ratio found for $\mathrm{GO}$ at room temperature and was thermally reduced was consistent with the theoretical value for $s p^{3}$ defects. We believe that our work makes an important contribution to understand the Raman spectrum of graphene oxides and we expect that the reported correlations could be used to obtain structural information of graphene oxide in situations where it is not possible to analyze the chemical composition and structural defects by other techniques. On the other hand, we proposed a reproducible methodology based on staking to build heterostructures based on graphene oxide avoiding vacancies and grain boundaries. This methodology can be very useful to manufacture heterostructures based on reduced graphene oxide and h-BN for applications where a high thermal stability is needed.

Author Contributions: Conceptualization, M.M.V.; Methodology, M.M.V., D.L.-D., E.D., J.A.D.-N., and V.C.; Formal Analysis M.M.V. and D.L.-D.; Investigation, D.L.-D, V.C., J.A.D.-N., and M.D.M.; Resources, E.D. and M.M.V.; Writing-Original Draft Preparation, M.M.V.; Writing-Review and Editing, M.M.V., D.L.-D., E.D., J.A.D.-N., V.C., and M.D.M.; Funding Acquisition, E.D. and M.M.V.; All authors have contributed substantially to the work reported. All authors have read and agreed to the published version of the manuscript.

Funding: This research was funded by the European Regional Development Fund (ERDF), Junta de Castilla y León, (SA045U16 and SA256P18) and by MINECO (CTQ2016-78895-R and MAT2016-75955-C2-2-R).

Acknowledgments: D.L.L thanks ERDF and Junta de Castilla y León for his postdoc contract. Authors also thank to Jose Luis García Fierro (Instituto de Catálisis y Petroleoquímica, CSIC, 28049 Cantoblanco, Madrid, Spain) for XPS measurements.

Conflicts of Interest: The authors declare no conflict of interest.

\section{References}

1. Potts, J.R.; Dreyer, D.R.; Bielawski, C.W. Graphene-Based Polymer Nanocomposites. Polymer 2011, 52, 5-25. [CrossRef]

2. Prezioso, S.; Perrozzi, F.; Giancaterini, L.; Cantalini, C.; Treossi, E.; Palermo, V.; Nardone, M.; Santucci, S.; Ottaviano, L. Graphene Oxide as a Practical Solution to High. Sensitivity Gas. Sensing. J. Phys. Chem. C 2013, 117, 10683-10690. [CrossRef]

3. Loh, K.P.; Bao, Q.; Eda, G.; Chhowalla, M. Graphene oxide as a chemically tunable platform for optical applications. Nat. Chem. 2010, 2, 1015-1024. [CrossRef]

4. Liu, J.; Cui, L.; Losic, D. Graphene and graphene oxide as new nanocarriers for drug delivery applications. Acta Biomater. 2013, 9, 9243-9257. [CrossRef] 
5. Rodríguez-García, S.; Santiago, R.; López-Díaz, D.; Merchán, M.D.; Velázquez, M.M.; Fierro, J.L.G.; Palomar, J. Role of the Structure of Graphene Oxide Sheets on the $\mathrm{CO}_{2}$ Adsorption Properties of Nanocomposites Based on Graphene Oxide and Polyaniline or $\mathrm{Fe}_{3} \mathrm{O}_{4}$-Nanoparticles. ACS Sustain. Chem. Eng. 2019, 7, 12464-12473. [CrossRef]

6. Liu, X.; Ma, R.; Wang, X.; Ma, Y.; Yang, Y.; Zhuang, L.; Zhang, S.; Jehan, R.; Chen, J.; Wang, X. Graphene oxide-based materials for efficient removal of heavy metal ions from aqueous solution: A review. Environ. Pollut. 2019, 252, 62-73. [CrossRef]

7. Ali, I.; Basheer, A.A.; Mbianda, X.Y.; Burakov, A.; Galunin, E.; Burakova, I.; Mkrtchyan, E.; Tkachev, A.; Grachev, V. Graphene based adsorbents for remediation of noxious pollutants from wastewater. Environ. Int. 2019, 127, 160-180. [CrossRef] [PubMed]

8. Luo, D.; Zhang, G.; Liu, J.; Sun, X. Evaluation Criteria for Reduced Graphene Oxide. J. Phys. Chem. C 2011, 115, 11327-11335. [CrossRef]

9. Fernández-Merino, M.J.; Guardia, L.; Paredes, J.I.; Villar-Rodil, S.; Solís-Fernández, P.; Martínez-Alonso, A.; Tascón, J.M.D. Vitamin C Is an Ideal Substitute for Hydrazine in the Reduction of Graphene Oxide Suspensions. J. Phys. Chem. C 2010, 114, 6426-6432. [CrossRef]

10. Martín-García, B.; Velázquez, M.M.; Rossella, F.; Bellani, V.; Diez, E.; García Fierro, J.L.; Pérez-Hernández, J.A.; Hernández-Toro, J.; Claramunt, S.; Cirera, A. Functionalization of Reduced Graphite Oxide Sheets with a Zwitterionic Surfactant. ChemPhysChem 2012, 13, 3682-3690. [CrossRef]

11. Wang, X.; Zhi, L.; Müllen, K. Transparent, Conductive Graphene Electrodes for Dye-Sensitized Solar Cells. Nano Lett. 2008, 8, 323-327. [CrossRef]

12. Claramunt, S.; Varea, A.; López-Díaz, D.; Velázquez, M.M.; Cornet, A.; Cirera, A. The Importance of Interbands on the Interpretation of the Raman Spectrum of Graphene Oxide. J. Phys. Chem. C 2015, 119, 10123-10129. [CrossRef]

13. Rozada, R.; Paredes, J.I.; López, M.J.; Villar-Rodil, S.; Cabria, I.; Alonso, J.A.; Martínez-Alonso, A.; Tascón, J.M.D. From graphene oxide to pristine graphene: Revealing the inner workings of the full structural restoration. Nanoscale 2015, 7, 2374-2390. [CrossRef]

14. Gupta, S.; Narayan, J. Reduced Graphene Oxide/Amorphous Carbon P-N Junctions: Nanosecond Laser Patterning. ACS Appl. Mater. Interfaces 2019, 11, 24318-24330. [CrossRef]

15. Pimenta, M.A.; Dresselhaus, G.; Dresselhaus, M.S.; Cancado, L.G.; Jorio, A.; Saito, R. Studying disorder in graphite-based systems by Raman spectroscopy. Phys. Chem. Chem. Phys. 2007, 9, 1276-1290. [CrossRef]

16. Malard, L.M.; Pimenta, M.A.; Dresselhaus, G.; Dresselhaus, M.S. Raman spectroscopy in graphene. Phys. Rep. 2009, 473, 51-87. [CrossRef]

17. Jia, X.; Campos-Delgado, J.; Terrones, M.; Meunier, V.; Dresselhaus, M.S. Graphene edges: A review of their fabrication and characterization. Nanoscale 2011, 3, 86-95. [CrossRef]

18. Jorio, A.; Dresselhaus, M.S.; Saito, R.; Dresselhaus, G. Raman Spectroscopy in Graphene Related Systems; Wiley-VCH Verlag GmbH \& Co.: Weinheim, Germany, 2011.

19. Ferrari, A.C.; Robertson, J. Interpretation of Raman spectra of disordered and amorphous carbon. Phys. Rev. B 2000, 61, 14095-14107. [CrossRef]

20. Ferrari, A.C.; Meyer, J.C.; Scardaci, V.; Casiraghi, C.; Lazzeri, M.; Mauri, F.; Piscanec, S.; Jiang, D.; Novoselov, K.S.; Roth, S.; et al. Raman Spectrum of Graphene and Graphene Layers. Phys. Rev. Lett. 2006, 97, 187401. [CrossRef]

21. Casiraghi, C.; Hartschuh, A.; Qian, H.; Piscanec, S.; Georgi, C.; Fasoli, A.; Novoselov, K.S.; Basko, D.M.; Ferrari, A.C. Raman Spectroscopy of Graphene Edges. Nano Lett. 2009, 9, 1433-1441. [CrossRef]

22. Cançado, L.G.; Jorio, A.; Ferreira, E.H.M.; Stavale, F.; Achete, C.A.; Capaz, R.B.; Moutinho, M.V.O.; Lombardo, A.; Kulmala, T.S.; Ferrari, A.C. Quantifying Defects in Graphene via Raman Spectroscopy at Different Excitation Energies. Nano Lett. 2011, 11, 3190-3196. [CrossRef] [PubMed]

23. Caridad, J.M.; Rossella, F.; Bellani, V.; Maicas, M.; Patrini, M.; Díez, E. Effects of particle contamination and substrate interaction on the Raman response of unintentionally doped graphene. J. Appl. Phys. 2010, 108, 084321. [CrossRef]

24. Caridad, J.M.; Rossella, F.; Bellani, V.; Grandi, M.S.; Diez, E. Automated detection and characterization of graphene and few-layer graphite via Raman spectroscopy. J. Raman Spectrosc. 2011, 42, 286-293. [CrossRef] 
25. Martins Ferreira, E.H.; Moutinho, M.V.O.; Stavale, F.; Lucchese, M.M.; Capaz, R.B.; Achete, C.A.; Jorio, A. Evolution of the Raman spectra from single-, few-, and many-layer graphene with increasing disorder. Phys. Rev. B 2010, 82, 125429. [CrossRef]

26. Szabó, T.; Berkesi, O.; Forgó, P.; Josepovits, K.; Sanakis, Y.; Petridis, D.; Dékány, I. Evolution of Surface Functional Groups in a Series of Progressively Oxidized Graphite Oxides. Chem. Mater. 2006, 18, 2740-2749. [CrossRef]

27. Kaniyoor, A.; Ramaprabhu, S. A Raman spectroscopic investigation of graphite oxide derived graphene. AIP Adv. 2012, 2, 032183. [CrossRef]

28. Wang, L.; Zhao, J.; Sun, Y.-Y.; Zhang, S.B. Characteristics of Raman spectra for graphene oxide from ab initio simulations. J. Chem. Phys. 2011, 135, 184503. [CrossRef]

29. Kudin, K.N.; Ozbas, B.; Schniepp, H.C.; Prud'homme, R.K.; Aksay, I.A.; Car, R. Raman Spectra of Graphite Oxide and Functionalized Graphene Sheets. Nano Lett. 2008, 8, 36-41. [CrossRef]

30. López-Díaz, D.; López Holgado, M.; García-Fierro, J.L.; Velázquez, M.M. Evolution of the Raman Spectrum with the Chemical Composition of Graphene Oxide. J. Phys. Chem. C 2017, 121, 20489-20497. [CrossRef]

31. Li, M.-Y.; Chen, C.-H.; Shi, Y.; Li, L.-J. Heterostructures based on two-dimensional layered materials and their potential applications. Mater. Today 2016, 19, 322-335. [CrossRef]

32. Lee, S.-M.; Kim, J.-H.; Ahn, J.-H. Graphene as a flexible electronic material: Mechanical limitations by defect formation and efforts to overcome. Mater. Today 2015, 18, 336-344. [CrossRef]

33. Mussa, Y.; Ahmed, F.; Arsalan, M.; Alsharaeh, E. Two dimensional (2D) reduced graphene oxide (RGO)/hexagonal boron nitride (h-BN) based nanocomposites as anodes for high temperature rechargeable lithium-ion batteries. Sci. Rep. 2020, 10, 1882. [CrossRef]

34. Hidalgo, R.S.; López-Díaz, D.; Velázquez, M.M. Graphene Oxide Thin Films: Influence of Chemical Structure and Deposition Methodology. Langmuir 2015, 31, 2697-2705. [CrossRef]

35. López-Díaz, D.; Merchán, M.D.; Velázquez, M.M.; Maestro, A. Understanding the Role of Oxidative Debris on the Structure of Graphene Oxide Films at the Air-Water Interface: A Neutron Reflectivity Study. ACS Appl. Mater. Interfaces 2020, in press.

36. Tuinstra, F.; Koenig, J.L. Raman Spectrum of Graphite. J. Chem. Phys. 1970, 53, 1126-1130. [CrossRef]

37. Vollebregt, S.; Ishihara, R.; Tichelaar, F.D.; Hou, Y.; Beenakker, C.I. Influence of the growth temperature on the first and second-order Raman band ratios and widths of carbon nanotubes and fibers. Carbon 2012, 50, 3542-3554. [CrossRef]

38. Pfeiffer, R.; Kuzmany, H.; Knoll, P.; Bokova, S.; Salk, N.; Günther, B. Evidence for trans-polyacetylene in nano-crystalline diamond films. Diam. Relat. Mater. 2003, 12, 268-271. [CrossRef]

39. Sadezky, A.; Muckenhuber, H.; Grothe, H.; Niessner, R.; Pöschl, U. Raman microspectroscopy of soot and related carbonaceous materials: Spectral analysis and structural information. Carbon 2005, 43, 1731-1742. [CrossRef]

40. Sze, S.K.; Siddique, N.; Sloan, J.J.; Escribano, R. Raman spectroscopic characterization of carbonaceous aerosols. Atmos. Environ. 2001, 35, 561-568. [CrossRef]

41. Dippel, B.; Heintzenberg, J. Soot characterization in atmospheric particles from different sources by NIR FT Raman spectroscopy. J. Aerosol Sci. 1999, 30, S907-S908. [CrossRef]

42. Shen, Y.; Yang, S.; Zhou, P.; Sun, Q.; Wang, P.; Wan, L.; Li, J.; Chen, L.; Wang, X.; Ding, S.; et al. Evolution of the band-gap and optical properties of graphene oxide with controllable reduction level. Carbon 2013, 62, 157-164. [CrossRef]

43. Cuesta, A.; Dhamelincourt, P.; Laureyns, J.; Martinez-Alonso, A.; Tascon, J. Comparative performance of X-ray diffraction and Raman microprobe techniques for the study of carbon materials. J. Mater. Chem. 1998, 8, 2875-2879. [CrossRef]

44. Ma, B.; Rodriguez, R.D.; Ruban, A.; Pavlov, S.; Sheremet, E. The correlation between electrical conductivity and second-order Raman modes of laser-reduced graphene oxide. Phys. Chem. Chem. Phys. 2019, 21, 10125-10134. [CrossRef] [PubMed]

45. Venezuela, P.; Lazzeri, M.; Mauri, F. Theory of double-resonant Raman spectra in graphene: Intensity and line shape of defect-induced and two-phonon bands. Phys. Rev. B 2011, 84, 035433. [CrossRef]

46. Lucchese, M.M.; Stavale, F.; Ferreira, E.H.M.; Vilani, C.; Moutinho, M.V.O.; Capaz, R.B.; Achete, C.A.; Jorio, A. Quantifying ion-induced defects and Raman relaxation length in graphene. Carbon 2010, 48, 1592-1597. [CrossRef] 
47. Eckmann, A.; Felten, A.; Mishchenko, A.; Britnell, L.; Krupke, R.; Novoselov, K.S.; Casiraghi, C. Probing the Nature of Defects in Graphene by Raman Spectroscopy. Nano Lett. 2012, 12, 3925-3930. [CrossRef] [PubMed]

48. Del Corro, E.; Taravillo, M.; González, J.; Baonza, V.G. Raman characterization of carbon materials under non-hydrostatic conditions. Carbon 2011, 49, 973-979. [CrossRef]

49. Ye, S.; Feng, J. The effect of sonication treatment of graphene oxide on the mechanical properties of the assembled films. RSC Adv. 2016, 6, 39681-39687. [CrossRef]

50. Masini, L.; Cecchini, M.; Girardo, S.; Cingolani, R.; Pisignano, D.; Beltram, F. Surface-acoustic-wave counterflow micropumps for on-chip liquid motion control in two-dimensional microchannel arrays. Lab Chip 2010, 10, 1997-2000. [CrossRef]

51. Castellanos-Gomez, A.; Buscema, M.; Molenaar, R.; Singh, V.; Janssen, L.; van der Zant, H.S.J.; Steele, G.A. Deterministic transfer of two-dimensional materials by all-dry viscoelastic stamping. 2D Mater. 2014, 1, 011002. [CrossRef]

52. Clericò, V.; Delgado-Notario, J.A.; Saiz-Bretín, M.; Malyshev, A.V.; Meziani, Y.M.; Hidalgo, P.; Méndez, B.; Amado, M.; Domínguez-Adame, F.; Diez, E. Quantum nanoconstrictions fabricated by cryo-etching in encapsulated graphene. Sci. Rep. 2019, 9, 13572. [CrossRef] [PubMed]

53. Souibgui, M.; Ajlani, H.; Cavanna, A.; Oueslati, M.; Meftah, A.; Madouri, A. Raman study of annealed two-dimensional heterostructure of graphene on hexagonal boron nitride. Superlattices Microstruct. 2017, 112, 394-403. [CrossRef]

(C) 2020 by the authors. Licensee MDPI, Basel, Switzerland. This article is an open access article distributed under the terms and conditions of the Creative Commons Attribution (CC BY) license (http://creativecommons.org/licenses/by/4.0/). 